\title{
ARTICLE
}

\section{Adult attention-deficit hyperactivity disorder and bipolar disorder}

\author{
Andrew Gleason \& David Castle
}

\begin{abstract}
Andrew Gleason is a senior registrar at Royal Melbourne Hospital, Australia. David Castle is Chair of Psychiatry at St Vincent's Hospital,

Melbourne, and the University of Melbourne, and a consultant psychiatrist at St Vincent's Hospital, Melbourne, Australia. Correspondence Dr Andrew Gleason, Neuropsychiatry Unit, Royal Melbourne Hospital, VIC 3050, Australia. Email: andrew.gleason@ mh.org.au
\end{abstract}

\begin{abstract}
SUMMARY
Increasing attention is being paid to the concept of attention-deficit hyperactivity disorder (ADHD) in adults, in concord with evidence that suggests a proportion of children with ADHD continue to manifest symptoms into adulthood. Attentiondeficit hyperactivity disorder has some symptoms in common with hypomania, and can co-occur with bipolar disorder. The diagnosis and management of ADHD in adults with bipolar disorder can be complicated, owing to challenges resulting from symptom overlap, questions of diagnostic validity and a paucity of empirical evidence to guide treatment. This article addresses comorbid ADHD and bipolar disorder, and provides practical suggestions for diagnosis and management.
\end{abstract}

\section{DECLARATION OF INTEREST}

D.C. has received fees from major pharmaceutical companies as a board member and for consultancy, grants and lectures.

A syndrome of hyperactivity and poor attention has been described in children for over a century and has evolved into the construct of attention-deficit hyperactivity disorder (ADHD) (Janakiraman 2010). Although traditionally viewed as affecting only children and adolescents, there is a growing consensus that some children and adolescents with ADHD may continue to manifest symptoms into adulthood (Asherson 2010; Moncrieff 2010). Estimates of this proportion vary from 4 to $66 \%$ (Janakiraman 2010) and prevalence rates of ADHD among adults in the general population are as high as 5\% (Klassen 2010). Attention-deficit hyperactivity disorder that continues into adulthood is associated with a high rate of psychiatric comorbidity, with anxiety disorders, substance misuse, unipolar depression and bipolar disorder being particularly common (Klassen 2010). ${ }^{\dagger}$

Readers may be interested in in Advances: Crimlisk H (2011) Developing integrated mental health services for adults with ADHD, 17: 461-469; Janakiraman R, Benning T (2010) Attention-deficit hyperactivity disorder in adults, 16: 96-104.

\section{Comorbid ADHD and bipolar disorder in adults}

Comorbid ADHD and bipolar disorder has been the subject of numerous studies - and much controversy - in children, yet little attention has been paid in adults in spite of apparently high rates of co-occurrence. Some studies suggest that ADHD is overrepresented in individuals with bipolar disorder (odds ratio of 8.3), and that up to 9-35\% of adults with bipolar disorder are affected (Klassen 2010; Parker 2010). The corollary of this is that comorbid ADHD and bipolar disorder may be underdiagnosed and undertreated (Klassen 2010).

There are several potential explanations for co-occurrence of ADHD and bipolar disorder in adults, none of which are mutually exclusive and a number of which may operate simultaneously to contribute to comorbidity. One possibility is that this finding is an artefact of symptom overlap, yet comorbid ADHD and bipolar disorder appears to be more common than would be expected by chance even when overlapping DSM-IV criteria are removed (Milberger 1995). Another possibility is Berkson's bias, where treatment-seeking patients tend to present with more comorbidities than the general affected population. It is also plausible that a common diathesis leaves patients vulnerable to both conditions, but the current neurobiological understanding of bipolar disorder and ADHD is insufficient to confirm or deny this possibility (Hegerl 2010; Klassen 2010).

A further dimension to the interplay of comorbidity is evidence for cognitive changes in bipolar disorder, which could mimic ADHD, resulting in more extensive symptom overlap than with current DSM (American Psychiatric Association 1994) or ICD (World Health Organization 1992) criteria alone. In particular, a sustained attention deficit is present early in the course of bipolar disorder, even during euthymia, and tends to become more prominent with repeated episodes of illness (Clark 2004). Additionally, deficits in working memory, verbal fluency and executive functions (such as planning, cognitive flexibility and cognitive control) occur (Soreca 2009). It is hypothetically possible that some of these symptoms could present early as a prodrome, in the form of childhood $\mathrm{ADHD}$, or that they could masquerade as ADHD in an adult (Klassen 2010).

When considering co-occurrence of conditions and symptom overlap, it is helpful to bear in mind 
that current criterion-based psychiatric diagnoses (i.e. those of DSM and ICD as they presently stand) are largely working hypotheses that facilitate standardised communication for research and clinical practice, rather than entities with a single definable pathophysiology and aetiology (Andreasen 2007; Mullen 2007). It is likely that the definitions of ADHD and bipolar disorder will change in the future as the understanding of their aetiology improves, and that this will also lead to a better understanding of the reasons for co-occurrence of these two conditions as they are currently conceptualised.

\section{The clinical course of comorbid ADHD and bipolar disorder}

Adults with comorbid ADHD and bipolar disorder have a more severe course of illness compared with those with either condition alone (Klassen 2010). There are also high rates of comorbidity with other psychiatric disorders, most notably anxiety disorders and substance misuse (Nierenberg 2005; Wingo 2007). Comorbid ADHD and bipolar disorder compared with bipolar disorder alone is associated with lower social functioning (Sentissi 2008), a lower age at first affective episode (Sachs 2000), more frequent affective episodes (especially depression) (Tamam 2006; Ryden 2009), more severe affective symptoms, a shorter duration of wellness, lower education, fewer relationships, more suicide attempts and more legal problems (Klassen 2010). It is thought that symptoms of ADHD, such as difficulty with focusing, concentration and memory, are often falsely attributed to bipolar disorder and hence left untreated in people with both conditions, and that on the whole, treatment of co-occurring ADHD and bipolar disorder is more often suboptimal compared with ADHD or bipolar disorder alone (Klassen 2010). In such cases, ADHD symptoms could potentially contribute to inter-episode functional impairment. Another possible factor contributing to the worse outcome in people with ADHD and bipolar disorder is that forgetfulness and inattention related to ADHD could potentially result in lower adherence to treatment, but this is unlikely to be the sole factor.

\section{Assessment}

The first priority when treating someone with presumed bipolar disorder is to establish that the patient has true bipolar disorder by taking a thorough history, and performing a physical examination and investigations as appropriate (Parker 2010). Once alternative primary conditions have been excluded, one should consider whether only one condition is present, i.e. whether suggested ADHD might just be a manifestation of bipolar disorder (Parker 2010). One should also screen for other comorbid conditions such as anxiety disorders and substance misuse, which are common in both bipolar disorder and ADHD: 65\% of people with ADHD and bipolar disorder have a lifetime history of at least one anxiety disorder (Klassen 2010). In patients who have symptoms of possible hypomania and who have never had a clear-cut manic episode, affective instability due to a personality disorder should also be considered in the differential diagnosis.

Once it has been established that the patient has bipolar disorder, a thorough assessment for ADHD can be conducted (Boxes 1 and 2). The manifestations of ADHD in adults and suggestions for assessment have been described in this journal (Janakiraman 2010) and elsewhere (Trollor 1999). Medical, psychiatric, social, education and vocational history should be sought. Retrospective information about childhood may not be accurate and reliable, so it is important to obtain medical records and school records, wherever possible, to verify childhood ADHD symptoms. Collateral history from parents, employers, significant others and carers should be obtained. Once childhood onset, chronicity and pervasiveness of symptoms have been established, one should enquire about the degree of functional impairment in life activities, including employment and interpersonal interactions (Murphy 2004).

\section{BOX 1 Diagnosing ADHD in adults}

1 Clinical interview to assess current ADHD symptoms (within the past 6 months)

2 Assess functional impairments at work, home and in relationships

3 Developmental history and childhood history of ADHD symptoms

4 Rule out other psychiatric disorders or establish comorbid conditions

5 Family history of psychiatric disorders (e.g. ADHD, obsessive-compulsive disorder, tics, intellectual disability, attention/concentration difficulties)

6 Complete physical examination (rule out head trauma, seizures, substance misuse, and hormonal problems)

7 Rule out ongoing social stressors as generators of symptoms that mimic ADHD

8 Neuropsychological assessments (e.g. attention, memory, intellectual functioning and academic achievement)

(Janakiraman 2010) 
BOX 2 Key questions in identifying ADHD

- Are the core symptoms - inattention, hyperactivity and impulsivity - clearly present?

- Does objective evidence show that the symptoms cause significant impairment in school, work or social domains and in daily adaptive functioning?

- Have symptoms been observed as part of the person's behaviour across situations since childhood?

-What evidence is there that symptoms are not due to lack of effort, poor vocational match, or transient situational or environmental circumstances?

- Are symptoms better explained by another psychiatric or medical diagnosis?

- Might other psychiatric conditions coexist with ADHD symptoms?

(Murphy 2004)

\section{Differential diagnosis}

It is important to consider that many who report distractibility, poor concentration or problems at school do not have ADHD (Carlat 2007). It is also unlikely that an adult who reports no problems with schoolwork or attention as a child has ADHD (Carlat 2007). Alternative diagnoses such as depression, a mixed episode, anxiety disorders, substance misuse and personality disorder need to be considered. Inattention or poor concentration commonly occur in depression and mixed states, together with the usual features of these syndromes. It can also be helpful to ask whether the patient usually has trouble with concentration when they are well, and if this has been continuous since childhood. Most people who report that their concentration normalises with their emotional state do not have ADHD (Carlat 2007). Medical

\section{BOX 3 Differential diagnoses of adult ADHD}

\section{Medical}

- Past head injury or anoxia

- Sleep disorders (e.g. sleep apnoea)

- Recent viral infection, including HIV

- Long-term medical illness (e.g. renal or liver failure)

- Seizure disorder (e.g. petit man

- Endocrine disorder (e.g. hypothyroidism, hyperthyroidism, hypoglycaemia)

Psychiatric

- Anxiety disorder

- Major depression
- Bipolar disorder (e.g. chronic hypomania)

- Cyclothymia

- Antisocial and borderline personality disorders

- Effect of prescribed medications (e.g. benzodiazepines, anticholinergic drugs, anticonvulsants)

- Other central nervous system disorder (e.g. degenerative disorders)

- Substance misuse (alcohol, long-term cocaine or amphetamine use, cannabis)

(Trollor 1999) and psychiatric differential diagnoses for ADHD are shown in Box 3.

Adults with ADHD present with slightly different characteristics than children. Hyperactive symptoms tend to decrease with age and adults manifest fewer externalising symptoms (Klassen 2010). Symptoms may take the form of:

- hyperactivity, which may be reported as 'nervousness'; symptoms can also be verbal, such as excessive talking;

- impulsivity, which may present as a tendency to interrupt others in social interactions, aggressive driving, impulsive spending, or the commencement of multiple projects, none of which are finished;

- poor attention, manifesting as careless mistakes, impaired planning, poor attention to detail or failure to listen when spoken to; this may also be reported as trouble concentrating (e.g. watching television, reading books);

- mood can be very reactive, and fluctuations can last from hours to a few days.

These symptoms are associated with psychosocial and occupational problems. Adults with ADHD are twice as likely to be arrested, have a twofold increased risk of divorce, a 78\% greater risk of being unemployed, and accumulate more speeding tickets than the general population (Janakiramann 2010; Klassen 2010).

Diagnostic complexities can arise given that there are a number of shared symptoms between ADHD and hypomania/mania (Table 1). Differentiating ADHD from bipolar II disorder, bipolar-spectrum disorders and episodes with mixed symptomatology can be difficult, as irritability, excessive activity, talkativeness, distractability, increased activity, physical restlessness, impulsive behaviour and poor judgement can be caused by ADHD (Klassen 2010). Nonetheless, many of the core symptoms of classical mania and hypomania (delusions of grandeur, euphoria, decreased need for sleep, racing thoughts, hypersexuality and psychotic symptoms) are not present in ADHD alone (Scheffer 2007).

There are a few key characteristics that assist in determining whether ADHD is present in someone with bipolar disorder. The most obvious contrasting features of ADHD and bipolar disorder lie in their time of onset and longitudinal course. Attention-deficit hyperactivity disorder has an early onset, usually occurring before the age of 7 , whereas a childhood onset of bipolar disorder is rare (Parker 2010). Attention-deficit hyperactivity disorder is pervasive such that patients usually report continuous symptoms, whereas bipolar disorder tends to be episodic (Parker 2010). 


\begin{tabular}{|c|c|c|}
\hline & ADHD & Mania/hypomania \\
\hline \multirow[t]{5}{*}{$\begin{array}{l}\text { Overlapping symptoms/ } \\
\text { signs }\end{array}$} & $\begin{array}{l}\text { Often talks excessively } \\
\text { Often blurts out answers before questions have been completed }\end{array}$ & More talkative than usual/pressure to keep talking \\
\hline & Is often easily distracted by extraneous stimuli & Distractibility \\
\hline & $\begin{array}{l}\text { Difficulty sustaining attention } \\
\text { Fails to give close attention to details/makes careless mistakes }\end{array}$ & $\begin{array}{l}\text { Sustained attention deficit (present during euthymia, worse in } \\
\text { mania/hypomania) (Clark 2004) }\end{array}$ \\
\hline & $\begin{array}{l}\text { Often fidgets with hands or feet } \\
\text { Has difficulty remaining seated } \\
\text { Runs or climbs about inappropriately/subjective restlessness } \\
\text { Has difficulty engaging in leisure activities quietly } \\
\text { Is often on the go as if driven by a motor }\end{array}$ & Psychomotor agitation \\
\hline & $\begin{array}{l}\text { Often interrupts or intrudes on others } \\
\text { Experiences difficulty awaiting one's turn }\end{array}$ & Loss of normal social inhibitions \\
\hline \multirow[t]{2}{*}{ Contrasting characteristics } & Onset in childhood; present before age 7 & Onset typically in early adulthood \\
\hline & Longstanding and temporally pervasive symptoms & $\begin{array}{l}\text { Cyclical (although inter-episode symptoms and dysfunction can } \\
\text { occur) (Judd 2002, 2003; Clark 2004) }\end{array}$ \\
\hline $\begin{array}{l}\text { Non-overlapping } \\
\text { symptoms }\end{array}$ & $\begin{array}{l}\text { Often avoids, dislikes or is reluctant to engage in tasks that } \\
\text { require sustained mental effort }\end{array}$ & $\begin{array}{l}\text { Inflated self-esteem/grandiosity (although this can also occur as } \\
\text { a defence mechanism in ADHD) (Gabbard 2005) } \\
\text { Increase in goal-directed activity } \\
\text { Flight of ideas or subjective experience of racing thoughts } \\
\text { Decreased need for sleep } \\
\text { Excessive involvement in pleasurable activities that have a high } \\
\text { potential for adverse consequences }\end{array}$ \\
\hline
\end{tabular}

Adapted from Klassen (2010).

Regarding age at onset, a history of ADHD can be established when school records, collateral history and the history taken from the patient support this diagnosis in childhood and before the onset of symptoms of mania or hypomania. One would also expect to see continuing symptoms of ADHD in someone with both ADHD and bipolar disorder in the absence of episodes of hypomania, mania or depression. This can be somewhat more complicated than it might initially seem, as recent studies have challenged the traditional view that bipolar disorder is purely episodic. In longitudinal studies over a period of years, people with bipolar disorder experienced symptoms for about half of follow-up weeks, but these were predominantly depressive rather than manic, and were mostly subsyndromal (Judd 2002, 2003). Additionally, attention deficits can occur in bipolar disorder even during euthymia, as discussed above (Clark 2004). These factors being taken into consideration, subsyndromal affective symptoms and bipolar disorder-related attention deficits are unlikely to be present continuously from childhood in the pattern typical of ADHD.

With respect to the differential diagnosis for a depressive presentation, it is helpful to consider that ADHD can be associated with a degree of dysphoria as a consequence of frustration with failures in life. Dysphoria arising from dismay over the psychosocial impact of ADHD symptoms is more likely to respond to ADHD treatment than to antidepressants, and can be distinguished from depression based on the absence of psychomotor changes, fatigue, sleep changes, anhedonia and suicidality, which are common in depression but not ADHD (Scheffer 2007).

\section{Rating scales}

There are a number of rating scales for ADHD in adults, including the Conners' Adult ADHD Rating Scale, the Brown Attention-Deficit Disorder Scale for Adults, the Wender Utah Rating Scale, the ADHD Rating Scales, the Current Symptoms Scale, and the Adult ADHD Self-Report Scale (Murphy 2004). These can be helpful as an adjunct to a thorough clinical assessment and in monitoring response to treatment, but have significant limitations. Self-report scales have questionable reliability, and no rating scale alone provides sufficient evidence to make a diagnosis of ADHD, as false positives may occur (Murphy 2004). No current scales have been extensively evaluated in people with comorbid ADHD and bipolar disorder. Neuropsychological testing may be of use, both in assessing impairments associated with ADHD and in those who may have neuropsychological deficits from other causes.

\section{Treatment}

\section{General principles}

There is currently a lack of empirical data to guide the treatment of ADHD in association with bipolar disorder, so patients should be treated 
on a case-by-case basis. We suggest a cautious approach. Clear-cut outcomes for treatment should be determined and monitored rather than trialling medication without targeting specific symptoms. When diagnoses are unequivocal and there is clear functional impairment, pharmacotherapy is justified. If a diagnosis is less clear-cut and functional impairment is less severe, a trial of psychosocial treatment with close monitoring may be a more reasonable approach.

Generally speaking, there are two broad approaches to managing independent comorbid conditions: sequential and hierarchical. The sequential approach involves identifying and managing the first temporal condition, and then managing any conditions that remain. The hierarchical approach involves determining the most clinically important factor, managing it, and then considering whether the less important condition has been corrected, or remains and requires management (Parker 2010).

The risks of untreated bipolar disorder are generally more severe than those of untreated $\mathrm{ADHD}$, so we recommend adopting a hierarchical approach and treating bipolar disorder first (Box 4). In some cases, symptoms that appear to be due to ADHD may respond to bipolar disorder treatment, making additional treatment for ADHD unnecessary. Bipolar disorder treatment should encompass medication, education, psychological and social interventions, with the aim of full control of symptoms where possible (Parker 2010).

BOX 4 Steps in treatment of presumed concurrent ADHD and bipolar disorder

1 Confirm the diagnosis/diagnoses through a good history (including collateral information), mental state exam, physical examination and appropriate investigations

2 Commence or optimise treatment for bipolar disorder (psychological and pharmacological); treat substance misuse if present; try to stage pharmacotherapy rather than commence multiple agents simultaneously if possible

3 Reassess for remaining conditions; treat anxiety disorders

4 If ADHD symptoms remain, commence treatment

5 If bipolar disorder symptoms re-occur, check adherence for bipolar disorder medications and blood levels where relevant; optimise bipolar disorder medications and psychosocial interventions; cease ADHD medications where necessary

6 In individuals that do not improve with treatment, reevaluate the diagnoses
Once bipolar disorder has been treated, a hierarchical approach can then be taken with the treatment of remaining comorbidities, including ADHD, treating the most destabilising illness first and then continuing in a step-wise fashion to manage the remaining conditions (Scheffer 2007). Where possible, it is best to introduce new medications one at a time in order to better assess the effect of each one. Comorbid anxiety disorders should probably be treated before ADHD, as stimulants could potentially worsen these conditions. In individuals who misuse substances, it may be beneficial to commence treatment for this at the same time as treatment for ADHD and bipolar disorder. Treatment of substance misuse may improve ADHD and bipolar disorder symptoms, and ADHD treatment may reduce the severity of comorbid substance misuse (Klassen 2010).

\section{Pharmacotherapy}

One of the most significant challenges in managing comorbid ADHD and bipolar disorder is that it is not entirely clear what effect stimulants have on bipolar disorder. There are a number of case reports of stimulant-induced mania, yet other studies, albeit small and uncontrolled, suggest that stimulants do not usually worsen mania and may even have some antimanic effects (Hegerl 2010; Hensch 2010). For example, one retrospective review of 16 patients with bipolar disorder (only 5 of whom also had ADHD) showed that methylphenidate improved concentration without causing mania over a mean treatment duration of 14 months (Lydon 2006). One factor in these conflicting findings is that a manic switch can occur after many months of stability (Scheffer 2007), so trials with long follow-up periods are needed to elucidate the risk of stimulant-induced mania in those with ADHD and bipolar disorder. There is some evidence that bupropion reduces symptoms of ADHD and depression without causing mania (Wilens 2003).

Given the paucity and conflicting findings of the research available, it is probably best to use stimulants only in carefully selected patients to reduce comorbid ADHD symptoms, and to avoid using stimulants without a mood stabiliser or in those who are not strictly adherent to drug regimes. One should also be circumspect about stimulant use in people with comorbid anxiety disorders, as anxiety symptoms may be exacerbated by stimulants. The patient and their carers should be warned of the possibility of mania as a result of stimulant use, and made aware of early warning symptoms (Morriss 2004). If symptoms of mania occur, a break from the stimulant and 
reassessment of the mood stabiliser regimen should be undertaken (Scheffer 2007). Clearly, other agents that make the symptoms worse (e.g. antidepressants triggering mania) should also be ceased (Scheffer 2007). In situations where stimulants have a significant positive effect on ADHD symptoms but are causing breakthrough manic symptoms, possible strategies include increasing the mood stabiliser dose or prescribing short breaks from the stimulant on weekends (Scheffer 2007).

One should enquire about a personal or family history of cardiac disease before beginning a stimulant. Stimulants may induce or exacerbate cardiac arrhythmias and hypertension. An electrocardiogram should be considered prior to treatment, especially in those over 40 years (Newcorn 2007). Medication history should include over-the-counter stimulants and caffeine, as methylphenidate, amphetamines and atomoxetine can accentuate their effects (Newcorn 2007).

\section{Psychosocial treatments}

Psychosocial treatments should be used for every patient with comorbid ADHD and bipolar disorder. Psychotherapeutic approaches to bipolar disorder that have shown benefits include psychoeducation, cognitive-behavioural therapy, interpersonal and social rhythm therapy, and family therapy (Lauder 2010). There is emerging evidence for cognitivebehavioural therapy and meta-cognitive therapy for adults with ADHD (Knight 2008; Solanto 2010). Meta-cognitive therapy incorporates cognitive-behavioural principles and aims to change styles of thinking in order to promote the development of executive self-management skills. Although no studies on psychological treatments for combined ADHD and bipolar disorder have been published to our knowledge, it makes sense to utilise the psychological treatments that have been established for non-comorbid ADHD and bipolar disorder as appropriate to the patient's clinical state, needs and preferences. Psychological techniques may need to be adjusted for people with ADHD, using, for example, shorter and more frequent sessions. Neuropsychological testing can enable psychological work to be adjusted according to an individual's deficits.

A psychodynamic understanding may also be helpful. In an age with effective biological treatments and diagnostic criteria that focus on behavioural phenomena rather than on a person's mental experience, it has become easy to forget that symptoms frequently have meaning and occur as part of the person's life. People with ADHD often have an impaired ability to soothe themselves because their attention impairment has affected their ability to internalise and maintain comforting parental introjects (Gabbard 2005). Failure to meet parental and societal expectations can lead to feelings of inadequacy and incompetence, resulting in the development of a defensive grandiosity, which could be confused with mania (Gabbard 2005). Adults with ADHD may fail to recognise interpersonal cues, and can develop stereotypical ways of relating to others as a defence against anxiety in interpersonal interactions (Gabbard 2005). Exploring the mental experience of the patient, meaning and context can be helpful diagnostically and in tailoring a treatment plan to an individual; this can also have a therapeutic effect in itself.

\section{Conclusions}

Bipolar disorder with comorbid adult ADHD appears to be a more serious condition than bipolar disorder alone. Given that clear-cut information about how to assess and treat comorbid ADHD and bipolar disorder is not currently available, adopting a conservative strategy that recognises that stimulants may precipitate mania is probably the best approach at present. It seems unlikely that treatment for bipolar disorder would make ADHD worse, but pharmacological treatment for ADHD could potentially exacerbate bipolar disorder (albeit this risk might be overstated). Some patients with significant ADHD symptoms that remain after bipolar disorder treatment has been established may improve significantly with ADHD treatment, making the possible risks of treatment worth bearing in some circumstances. Future research is required to clarify these uncertainties and to shed light on the nature of comorbid adult $\mathrm{ADHD}$ and bipolar disorder.

\section{Acknowledgement}

We thank Dr Joel King for providing editorial assistance with the manuscript.

\section{References}

American Psychiatric Association (1994) Diagnostic and Statistical Manual of Mental Disorders (4th edn) (DSM-IV). APA.

Andreasen NC (2007) DSM and the death of phenomenology in America: an example of unintended consequences. Schizophrenia Bulletin 33: $108-12$.

Asherson P, Adamou M, Bolea B, et al (2010) Is ADHD a valid diagnosis in adults? Yes. BMJ 340: c549.

Carlat DJ (2007) My favorite tips for sorting out diagnostic quandaries with bipolar disorder and adult attention-deficit hyperactivity disorder. Psychiatric Clinics of North America 30: 233-8.

Clark L, Goodwin GM (2004) State- and trait-related deficits in sustained attention in bipolar disorder. European Archives of Psychiatry and Clinical Neuroscience 254: 61-8.
MCO answers

1 e 2 c 3 b 4 e $5 d$ 
Gabbard GO (2005) Psychodynamic Psychiatry in Clinical Practice. American Psychiatric Publishing.

Hegerl U, Himmerich H, Engmann B, et al (2010) Mania and attentiondeficit/hyperactivity disorder: common symptomatology, common pathophysiology and common treatment? Current Opinion in Psychiatry 23: $1-7$

Hensch T, Himmerich H, Hegerl U, et al (2010) Stimulants in bipolar disorder: beyond common beliefs. CNS Spectrums 15: 469-72.

Janakiraman R, Benning T (2010) Attention-deficit hyperactivity disorder in adults. Advances in Psychiatric Treatment 16: 96-104.

Judd LL, Akiskal HS, Schettler PJ, et al (2002) The long-term natural history of the weekly symptomatic status of bipolar I disorder. Archives of General Psychiatry 59: 530-7.

Judd LL, Akiskal HS, Schettler PJ, et al (2003) A prospective investigation of the natural history of the long-term weekly symptomatic status of bipolar II disorder. Archives of General Psychiatry 60: 261-9.

Klassen LJ, Katzman MA, Chokka P (2010) Adult ADHD and its comorbidities, with a focus on bipolar disorder. Journal of Affective Disorders 124: 1-8.

Knight LA, Rooney M, Chronis-Tuscano A (2008) Psychosocial treatments for attention-deficit/hyperactivity disorder. Current Psychiatry Reports 10: 412-8.

Lauder SD, Berk M, Castle DJ, et al (2010) The role of psychotherapy in bipolar disorder. Medical Journal of Australia 193: S31-5.

Lydon E, El-Mallakh RS (2006) Naturalistic long-term use of methylphenidate in bipolar disorder. Journal of Clincal Psychopharmacology 26: 516-8.

Milberger S, Biederman J, Faraone SV, et al (1995) Attention deficit hyperactivity disorder and comorbid disorders: issues of overlapping symptoms. American Journal of Psychiatry 152: 1793-9.

Moncrieff J, Timimi S (2010) Is ADHD a valid diagnosis in adults? No. BMJ 340: 0547 .

Morriss $\mathrm{R}$ (2004) The early warning symptom intervention for patients with bipolar affective disorder. Advances in Psychiatric Treatment 10 $18-26$.

Mullen PE (2007) A modest proposal for another phenomenological approach to psychopathology. Schizophrenia Bulletin 33: 113-21.

Murphy KR, Adler LA (2004) Assessing attention-deficit/hyperactivity disorder in adults: focus on rating scales. Journal of Clinical Psychiatry 65 (suppl 3): 13-7.
Newcorn JH (2007) Age-related considerations in the treatment of ADHD. CNS Spectrums 12 (suppl 23): 10-3.

Nierenberg AA, Miyahara S, Spencer T, et al (2005) Clinical and diagnostic implications of lifetime attention-deficit/hyperactivity disorder comorbidity in adults with bipolar disorder: data from the first 1000 STEPbipolar disorder participants. Biological Psychiatry 57: 1467-73.

Parker GB (2010) Comorbidities in bipolar disorder: models and management. Medical Journal of Australia 293: S18-20.

Ryden E, Thase ME, Straht D, et al (2009) A history of childhood attention-deficit hyperactivity disorder (ADHD) impacts clinical outcome in adult bipolar patients regardless of current ADHD. Acta Psychiatrica Scandinavica 120: 239-46.

Sachs GS, Baldassano CF, Truman CJ, et al (2000) Comorbidity of attention deficity hyperactivity disorder with early- and late-onset bipolar disorder. American Journal of Psychiatry 157: 466-8.

Scheffer RE (2007) Concurrent ADHD and bipolar disorder. Current Psychiatry Reports 9: 415-9.

Sentissi 0, Navarro JC, Oliveira HD, et al (2008) Bipolar disorders and quality of life: the impact of attention deficit/hyperactivity disorder and substance abuse in euthymic patients. Psychiatry Research 161: 36-42.

Solanto MV, Marks DJ, Wasserstein J, et al (2010) Efficacy of metacognitive therapy for adult ADHD. American Journal of Psychiatry 167 : $958-68$.

Soreca I, Frank E, Kupfer KJ (2009) The phenomenology of bipolar disorder: what drives the high rate of medical burden and determines long-term prognosis? Depression and Anxiety 26: 73-82

Tamam L, Tuglu C, Karatas G, et al (2006) Adult attention-deficit hyperactivity disorder in patients with bipolar I disorder in remission: preliminary study. Psychiatry and Clinical Neurosciences 60: 480-5.

Trollor JN (1999) Attention deficit hyperactivity disorder in adults: conceptual and clinical issues. Medical Journal of Australia 171: $421-5$.

Wilens TE, Prince JB, Spencer T, et al (2003) An open trial of bupropion for the treatment of adults with attention-deficit/hyperactivity disorder and bipolar disorder. Biological Psychiatry 54: 9-16.

Wingo AP, Ghaemi SN (2007) A systematic review of rates and diagnositc validity of comorbid adult attention-deficit/hyperactivity disorder and bipolar disorder. Jounal of Clinical Psychiatry 68: 1776-84.

World Health Organization (1992) Tenth Revision of the International Classification of Diseases and Related Health Problems (IDC-10). WHO.

\section{MCOs}

Select the single best option for each question stem

1 Which of the following have welldemonstrated sensitivity and specificity sufficient to make a diagnosis of ADHD in adults who have bipolar disorder:

a patient self-report of poor attention at primary school

b Conners' Adult ADHD Rating Scales

c Wender Utah Rating Scale

d Current Symptom Scale

e none of the above.

2 A university student with well-established bipolar disorder treated with lithium complains of 'trouble concentrating' that is affecting his ability to function academically. What is the least reasonable next step:

a assess him for signs and symptoms of mania/ hypomania b check his serum lithium level

c commence a stimulant

d screen for drug and alcohol misuse

e ask him to bring teachers' reports from primary school to the next appointment.

3 Grandiosity in a person with bipolar disorder and ADHD is least likely to be associated with:

a a psychological defence mechanism

b alcohol withdrawal

c hypomania/mania

$\mathrm{d}$ a personality disorder

e non-adherence to mood stabilisers.

4 Differential diagnosis for ADHD includes:

a past head injury

b epilepsy

c substance misuse/dependence

d major depression

e all of the above.
5 Based on current research, what proportion of adults with bipolar disorder is thought to also have ADHD:

a $0.1-1 \%$

b $2-4 \%$

c $5-8 \%$

d $9-35 \%$

e $36-50 \%$. 